\title{
CURRENT TOPIC
}

\section{Are we 'Fit for the Future' now?}

\author{
David M B Hall
}

These two papers mark the 20th anniversary of the publication of the report of the Royal Commission on Child Health Services entitled Fit for the Future.
Twenty years after Fit for the Future was published, ${ }^{1}$ Donald Court and his committee colleagues might justifiably be pleased with the progress made in many of the areas highlighted in the report, such as the care of the newborn, parent held records, child psychiatry services, the district handicap team, flexible training, and the possibility of progression to consultant status for some senior clinical medical officers (SCMOs). But they might be less satisfied with the progress made in implementing the report's priority recommendations, which incorporated four quite radical proposals: an integrated child health service; career changes for clinical medical officers (CMOs) and SCMOs, with increasing numbers of consultant community paediatricians (CCPs); general practitioner paediatricians (GPPs); and child health visitors. These were a response to what Court perceived as the most pressing issue in paediatrics: 'We urge the Government to reach a decision about the re-organisation and staffing of primary care services for children at the earliest possible moment ... [this] will end the uncertainty about the pre-school and school health services and the position of the doctors working in them' (page 365).

\section{The integrated child health service}

The hospital service, general practice, and the local authority network of child welfare and school health services grew up in parallel but each of the three had different aims, culture, staffing, and management. Several golden opportunities to unite these three services have been squandered. The Dawson report (1920) and the Sheldon report (1967) both proposed a rationalisation of the system but their suggestions were not implemented. The NHS Act of 1946 , influenced by the current assumption that prevention could and should be separated from treatment, enshrined the tripartite system in the NHS legislation. In 1973 the NHS Reorganisation Act removed all responsibility for medical services from local authorities and transferred this to 'autonomous' area health authorities within the NHS, providing yet another opportunity to review child health services. This reorganisation was the stimulus for the Court report.

Following this reorganisation, the area health authority took over responsibility for the local authority medical staff, mainly CMOs and SCMOs. Many of these had received little or no formal training; there was no proper career structure and no clinical or academic leadership. The CMOs and SCMOs provided a disparate network of community based services. Some of these were primary or 'first contact' services, whose aims were to prevent illness, promote good health and deal with minor problems; for example, infant welfare and feeding advice, immunisation, and child health surveillance. Some were secondary or referral services, since they needed specialist expertise, such as audiology and disability clinics, and support for social services in respect of child abuse. The school health service was an example of a programme with both primary and secondary care elements.

Court could not have foreseen the extent to which the effectiveness, efficiency, and quality of community services would soon come under scrutiny, but he did address the fundamental weaknesses of the system-the low academic status of preventive medicine, the uncomfortable mixture of primary and secondary care provided by the community network, the fact that CMOs and SCMOs were not allowed to prescribe treatment or initiate referrals to consultants in the hospital service, and the lack of continuity between preschool and school health services. He saw that these defects were confusing to parents, wasteful of resources, and frustrating to the staff. Two recurring themes in his writing were the artificial nature of the distinction between prevention and curative medicine, and research: 'the need, in the next 25 years, to give prevention the degree of scientific attention that has been given in the last 25 years to treatment' (page 90).

He suggested that the health care system for children should have only two parts, not three. All primary care aspects of community child health services were to be passed over to a new breed of general practitioner, the GPP. This person would undertake preventive care for all the children in the practice, ideally in a group practice setting. Up to $70 \%$ of their working week might be spent in such work but they would still continue with some adult practice. Support was to be provided within the primary care team by a child health visitor who would participate in curative work as well as prevention. Problems identified at primary care level would be referred as appropriate to consultant hospital or community paediatricians, all of whom would be full members of a unified department of child health within the hospital 
service. CMOs would have the opportunity to become GPPs or child health practitioners within the general practice framework. Many SCMOs (including some without the MRCP qualification) should be promoted to consultant status and others would be designated as a clinical specialists in paediatrics.

Sadly, the future for many CMOs is still uncertain and it took 10 years for Court's wise advice about SCMOs to be followed-only in the late 1980s was the paediatric committee of the Joint Committee on Higher Medical Training able to implement a scheme whereby SCMOs could be 'accredited' and eventually in 1993 an effort was made to resolve the remaining contractual and career development issues. ${ }^{2}$ The European Specialist Medical Qualifications Order and 'Calmanisation' offer us a last chance to tidy up this unsatisfactory situation. ${ }^{3}$

There are several reasons why it took so long to act on the main recommendations of the report. First, there were some real difficulties with Court's proposals. The distinction between primary and secondary care proved to be contentious and the costs were probably underestimated. Second, there were professional disagreements, both within and between disciplines, which hindered progress. However, by the late 1980 s there was an increasing consensus that primary care paediatrics belonged with primary care teams based in general practice and that doctors providing secondary care should be part of a unified paediatric department along with their hospital colleagues. The need for a more effective partnership between primary and secondary care was also accepted. The British Paediatric Association defined a combined service as one that unified community and hospital secondary services, and an integrated service as one that brought together primary and secondary care. ${ }^{4}$

The NHS and Community Care Act 1990 seemed to offer the long awaited opportunity finally to unite community and hospital paediatric services across the country. Some districts had already achieved this, overcoming many of the previous obstacles to service planning and creative deployment of resources. Some managers were disappointed when creation of these unified departments did not bring about miracles overnight, but unless attitudes and skills change as well as management structures, the consumer is unlikely to notice any immediate difference.

To the disappointment of many paediatricians, this latest opportunity has in many districts been squandered by political dogma. The concept of purchaser-provider separation had promised not just to preserve but also to strengthen the role of preventive medicine and chronic care, in the face of the resource hungry hospital services; however, there were doubts as to whether the purchasers had the teeth or the stomach to insist on such changes and a vocal minority of professionals voiced fears that the community service would be asset stripped if amalgamated with the hospital. This played into the hands of the government whose philosophy was that competition between community and hospital trusts would improve standards and drive down costs. While doctors increasingly were called upon to practice evidence-based medicine, politicians felt no such obligation and were unperturbed by the lack of evidence that such competition would result in savings or better services. ${ }^{5}$ Many districts now report unseemly rivalry between trusts who should be cooperating, to the irritation of the professionals and often to the embarrassment of managers, many of whom are genuinely more interested in good services than financial deals.

\section{The GPP}

The concept of the GPP never found wide acceptance, ${ }^{6}$ for a number of reasons. First, while many general practitioners were, and still are, committed to specialisation within the primary care team, the idea of spending the bulk of their time with just one group of patients, defined by age, was contrary to their general philosophy of caring for the family as a whole. Second, the numbers did not add up - the idea might have worked in a large group practice but was likely to present real problems in smaller practices. Third, there was much debate as to whether a GPP could reasonably be expected to reach the level of skill in developmental diagnosis or educational medicine claimed by the community child health doctors, since a practice population would be unlikely to generate enough paediatric problems to maintain the skills of a GPP.

After increasing doubts about the value of routine examinations of well children, a reappraisal of child health surveillance was initiated in 1986. This helped to resolve the question of where primary care ended and secondary care began. A core programme of routine child health checks, immunisation, and health promotion was established, ${ }^{7}$ and all parties agreed that this programme must be regarded as primary care. The new child health surveillance programme was incorporated into the new contract for general practice of 1990 which permitted general practitioners to be 'accredited' to carry out child health surveillance provided that they fulfilled certain criteria. A new fee scale was established, to encourage general practitioners to provide child health surveillance and to pursue a high immunisation uptake within their practice.

Child health surveillance and immunisation are now usually provided by general practitioners, though in very deprived areas where primary care services are struggling, child health clinics may still be needed. Health promotion and primary prevention are delivered largely by health visitors who work alongside their general practitioner colleagues. CMOs and SCMOs have taken on an increasing proportion of secondary care, in particular the expanding workload generated by child protection and emotional and behavioural disorder. Some CMOs have entered general practice but many fewer than Court had envisaged.

All this represents progress, but there is no room for complacency. The programme of child health surveillance has a rational basis, but its benefits in isolation are at best modest 
and provision of training in surveillance without a solid basis in paediatrics is of limited value. Many general practice trainees undertake a senior house officer post in paediatrics but spend too much time dealing with sick children on the wards and not enough learning about the problems that are common for the general practitioner. There are still insufficient senior house officer posts for all general practice trainees, with no immediate prospect of rectifying this situation. We need a carefully planned paediatric syllabus and training plan for every trainee, designed so that even those who cannot obtain a paediatric post can participate in structured training.

Community secondary care services are now well established, but there are many unsolved problems. Little is known about the functions of the 'district handicap team' and the child protection service, or the reasons for the differences between trusts in the standard and breadth of service provided. There is an impending manpower crisis in children's audiology services. We are still very bad at assessing the benefits of what we do. Information technology promised so much, but at present our computers ingest immense amounts of data at considerable expense, yet give little real information back. The description and analysis of specific learning disability has generated a vast literature; and the service needs of school age children and the place of the school health service have been reviewed recently. ${ }^{8}$ Nevertheless, research into the potential benefits of sophisticated 'educational medicine', the multidisciplinary assessment of children with problems affecting learning, is still virgin territory.

\section{Child health visitors}

'The main justification for the distinction between prevention and treatment has been that they cannot be combined without an unacceptable sacrifice of preventive work ... [but] this distinction is wasteful and confusing to the parents and underestimates the value to the family of having one familiar figure ... [for] health promotion and for illness' (page 108).

Health visitors are currently uncertain of their role. In addition to their traditional work with children and mothers, they are asked to undertake community development projects, specialist work such as tuberculosis contact tracing, and health promotion with adult men, the elderly, or the disabled. The value of their health promoting activities with children is being questioned. Court's analysis was that the health visitor was ideally placed to take on the care of sick children in the community, supported where necessary by a more specialised nurse based within the hospital department. Most of those who fiercely declare their faith in health promotion oppose Court's view. Nevertheless many health visitors would welcome the opportunity to undertake the care of sick children at home, with appropriate support; indeed, this already happens in many informal ways. The sad fact is that we still have little research data to help us decide whether his proposals would (in 1990s jargon) produce more 'health gain' than a purely health promoting model of health visiting.

\section{In conclusion}

The first law of ' $O$ ' level economics tells us that resources are, and always will be, scarce. Donald Court wrote hopefully of a future time when 'the financial crisis would be contained and there would once again be sustained growth in resources and public expenditure' (page 364) but he did not live to see this Utopian state of affairs and I doubt if I shall either.

Rereading Fit for the Future, I reflected that since 1976 the number of consultant paediatricians has increased more than threefold. Should investment in medical care continue to expand at such a rate? Can we make better use of existing resources? Is there a law of diminishing returns as we spend more and more on health care? Would we do better to invest our 'sustained growth in resources', if it ever comes, in housing and education rather than the health care system?

1 Court SDM. Fit for the future. Report of the Committee on Child Health Services. London: HMSO, 1976.

2 British Medical Association. Report of the foint Working Party on Medical Services for Children. London: BMA, 1993. on Medical

3 British Paediatric Association and Specialist Advisory British Paediatric Association and Specialist Advisory Training. Transition guidelines for non-consultant career-grade Training. Transition guidelines for non-consultant career-grade doctors. London: BPA, 1996.

4 British Paediatric Association. Towards a combined child health service. London: BPA, 1991.

5 Anonymous. Surgery needed-a survey of health care. The Economist 6 July 1991: 3-22 (suppl)

6 Department of Health. Child health in the community: a guide to good practice. London: Department of Health, 1996 21-2.

7 Hall DMB. Health for all children: report of the foint Working Party on Child Health Surveillance. Oxford: Oxford University Press, 1989 (1st Ed), 1996 (3rd Ed).

8 Polnay L. The health care needs of school aged children. London: British Paediatric Association, 1996.

\title{
Donald Court: man of vision (1912-94)
}

\author{
A W Craft
}

The 1970 s were a time of consolidation and reflection for paediatrics in the UK. Paediatrics had begun to develop as a separate specialty before the second world war and noteworthy landmarks were the formation of the British Paediatric Association (BPA) in 1928 and the establishment of the first full time professor of child health (James Spence) in England in Infirmary, Newcastle upon Tyne NE1 4LP

Correspondence to: Professor Craft.
1942 in Newcastle upon Tyne. Among the new group of young enthusiastic doctors who came into paediatrics after the war was Donald Court. In the 20 year postwar period the organisation of the care of children underwent a rapid expansion with paediatric units being set up in most major, and many smaller, hospitals. This growth had been largely uncoordi- 